\title{
Vulnerability or resilience of motopsin knockout mice to maternal separation stress depending on adulthood behaviors
}

This article was published in the following Dove Press journal:

Neuropsychiatric Disease and Treatment

\author{
Chiharu Hidaka ${ }^{1,2}$ \\ Taiki Kashio' \\ Daiju Uchigaki ${ }^{3}$ \\ Shinichi Mitsui ${ }^{1,3}$
}

'Department of Rehabilitation Sciences, Gunma University Graduate School of Health Sciences, Maebashi, Japan; ${ }^{2}$ Department of Neurobiology and Anatomy, Kochi Medical School, Kochi University, Nankoku, Japan; ${ }^{3}$ Department of Occupational Therapy, Gunma University, Maebashi, Japan

Correspondence: Shinichi Mitsui

Department of Rehabilitation Sciences, Gunma University Graduate School of Health Sciences, 3-39-22 Showa-machi, Maebashi 37I-85I4, Japan

Tel/fax +8I 272208950

Email smitsui@gunma-u.ac.jp
Background: Both environmental and genetic conditions contribute to the robust development of neuronal circuits and adulthood behaviors. Loss of motopsin gene function causes severe intellectual disability in humans and enhanced social behavior in mice. Furthermore, childhood maltreatment is a risk factor for some psychiatric disorders, and children with disabilities have a higher risk of abuse than healthy children.

Materials and methods: In this study, we investigated the effects of maternal separation (MS) on adulthood behaviors of motopsin knockout (KO) and wild-type (WT) mice.

Results: The MS paradigm decreased the duration that WT mice stayed in the center area of an open field, but not for motopsin KO mice; however, it decreased the novel object recognition index in both genotypes. In the marble burying test, motopsin $\mathrm{KO}$ mice buried fewer marbles than WT mice, regardless of the rearing conditions. The MS paradigm slightly increased and reduced open arm entry in the elevated plus maze by WT and motopsin KO mice, respectively. In the three-chamber test, the rate of sniffing the animal cage was increased by the MS paradigm only for motopsin KO mice. After the three-chamber test, motopsin KO mice had fewer cFos-positive cells in the prelimbic cortex, which is involved in emotional response, than WT mice. In the infralimbic cortex, the MS paradigm decreased the number of cFos-positive cells in motopsin KO mice.

Conclusion: Our results suggest that motopsin deficiency and childhood adversity independently affect some behaviors, but they may interfere with each other for other behaviors. Defective neuronal circuits in the prefrontal cortex may add to this complexity.

Keywords: early-life stress, mental retardation, neurotrypsin, PFC, PRSS12

\section{Introduction}

It has long been believed that both environmental and genetic conditions contribute to the robust development of neuronal circuits and adulthood behaviors. ${ }^{1-3}$ Disturbance of genetic or environmental conditions, such as gene mutations, childhood maltreatment, or drug addiction, increases the risk for developmental or psychiatric disorders. Defects in $>100$ genes account for early-onset intellectual disabilities, which the Diagnostic and Statistical Manual of Mental Disorders, fifth edition (DMS-5) defines as disorders with onset during the developmental period causing intellectual as well as adaptive deficits in conceptual, social, and practical areas. ${ }^{4,5}$ Among them, a 4 bp deletion in the motopsin (also called neurotrypsin or prss12) gene causes severe intellectual disability. ${ }^{6}$ Motopsin is activitydependently secreted from pyramidal neurons in the cerebral cortex and hippocampus and cleaves heparan sulfate proteoglycan, agrin. ${ }^{7,8}$ Motopsin-dependent cleavage of agrin is essential for the formation of filopodia, which is known to be a precursor structure of spines, a postsynaptic structure. ${ }^{9}$ Enhanced expression of motopsin in the abovementioned 
brain regions during the neonatal period suggests the importance of this protease in neurodevelopment. ${ }^{10,11}$ Indeed, the lack of motopsin functions causes abnormal behaviors accompanied with functional deficit in hippocampal neurons. ${ }^{12}$ In a Morris water maze test, motopsin knockout (KO) mice swim in the target quadrant in significantly less duration than wild-type (WT) mice. Although motopsin KO mice have an ability to discriminate between familiar and stranger mice, they spend significantly longer duration to sniff a familiar mouse than WT mice in social recognition and three-chamber tests. Motopsin KO mice show decreased spine density in hippocampal neurons. Morris water maze task and social interaction induce the phosphorylation of cAMP responsive element binding protein (CREB), which is known as a key event for neuronal plasticity, in hippocampal neurons of WT mice, whereas the level of CREB phosphorylation is markedly reduced in hippocampal neurons of motopsin $\mathrm{KO}$ mice. Hence, motopsin deficiency appears to disturb the development of neuronal circuits connected to the hippocampus.

In terms of environmental factors, childhood maltreatment is the leading risk factor for mental disorders such as depression, bipolar disorder, and substance and alcohol abuse. ${ }^{13,14}$ Childhood maltreatment alters the trajectories of brain development and neuronal circuits involved in emotional behaviors. ${ }^{15-17}$ It is mimicked by a maternal separation (MS) paradigm in rodent models, in which infants are removed daily from their dams for a few hours. MS paradigms impair myelination and neuronal activity in the adult prefrontal cortex (PFC). ${ }^{18,19}$ Additionally, many reports stated that MS paradigms cause long-lasting changes in anxiety-like, depression-like, and social behaviors in mice. ${ }^{20-22}$ Although a recent report found that children with disabilities are more frequently victims of abuse than healthy children, ${ }^{23}$ the effects of early-life adversity on pups with atypical neurodevelopment are poorly understood. In this study, we applied an MS paradigm to motopsin $\mathrm{KO}$ mice to examine its effects on adulthood behaviors.

\section{Materials and methods}

\section{Animals}

Motopsin homozygous KO mice and WT mice were obtained by breeding motopsin heterozygous mice with the C57BL/ 6 background. ${ }^{12}$ Mice were housed in transparent polycarbonate cages with wooden bedding under a 12-hour light/dark cycle (lights on at 6:00 am and lights off at 6:00 pm), with ad libitum access to food pellets and water. In this study, male pups were used as subjects. All animal experiments were approved by the Institutional Animal Experiment Committee at Gunma University according to the Regulation of Animal Experiment in Gunma University.

\section{Experimental design}

A schematic illustrating the experimental procedure is presented in Figure 1. Subject mice were randomly separated into two groups at postnatal day (P) 2. In one group (animal facility rearing [AFR]) as a control group, pups were raised undisturbedly except for changing the bedding. The other was the MS group in which an MS paradigm (described in detail below) was applied to pups from P2 until P14. At P27, pups were weaned and housed in groups (3-5 mice/cage) of same sex siblings. To reduce social effects, both AFR and MS mice were individually housed for 2 weeks before behavioral tests were started at 14 weeks old. We used two cohorts of subjects. The order of behavioral examinations was as

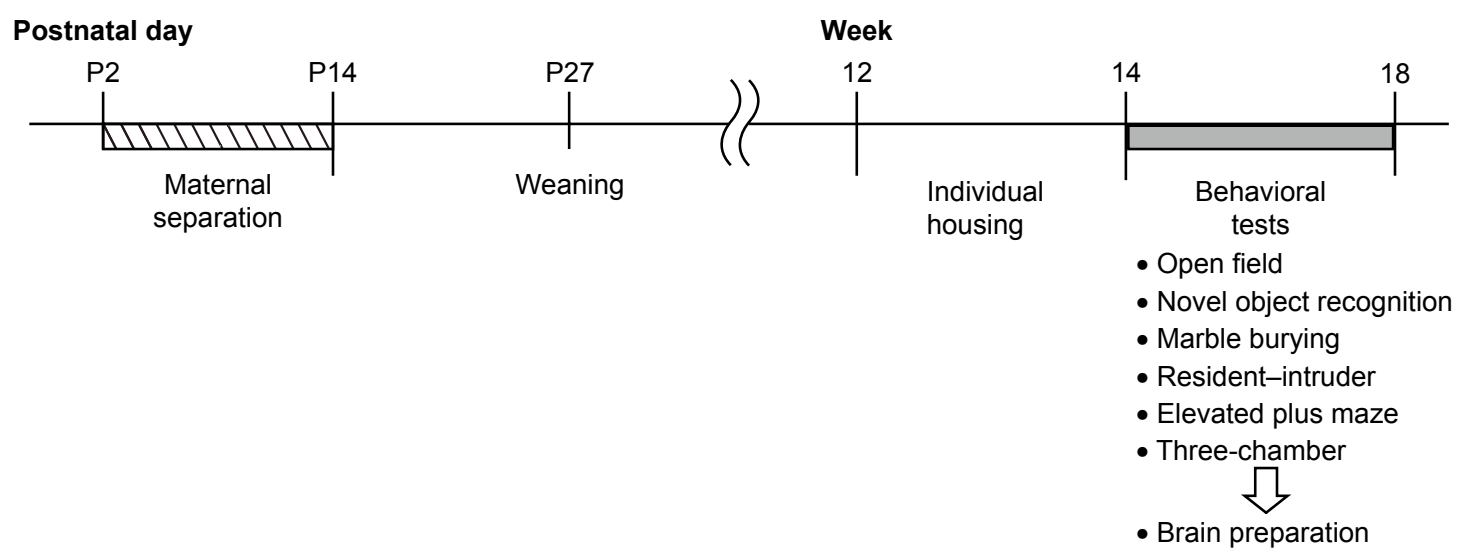

Figure I Schematic illustration of the experimental schedule.

Notes: Subjects were applied with a maternal separation paradigm between P2 and PI4 and weaned at P27. Before behavioral tests, they were individually housed for 2 weeks. Brains were prepared 3 hours after the three-chamber test.

Abbreviation: $\mathrm{P}$, postnatal day. 
follows: Cohort 1 (AFR-WT, n=4; MS-WT, n=4; AFR-KO, $\mathrm{n}=9$; and $\mathrm{MS}-\mathrm{KO}, \mathrm{n}=5$ ): an open field test followed by a novel object recognition test, a marble burying test, a residentintruder test, an elevated plus maze test, and three-chamber tests with a 2- to 8-day interexamination interval; Cohort 2 (AFR-WT, n=4; MS-WT, n=6; AFR-KO, n=3; and MS-KO, $\mathrm{n}=8$ ): an open field test followed by a novel object recognition test, an elevated plus maze test, a resident-intruder test, a marble burying test, three-chamber tests with a 1- to 3-day interexamination interval. All behavioral examinations were carried out during the dark period (9:30 pm-3:00 am). Brains were prepared from subjects 3 hours after the three-chamber test was completed.

\section{MS procedure}

MS was performed with modifications according to a previous report. ${ }^{24}$ Dams were individually housed in a standard cage $(20 \times 12 \times 15 \mathrm{~cm}) 2$ weeks after mating, and parturition was monitored every evening. The litter was culled to 7-9 pups per dam at P2. The whole litter in the MS group was transferred to a new cage for 3 hours in the dark phase (9:30 pm-0:30 am). The MS paradigm was applied daily from P2 to P14. After the separation, pups were returned to nests and reunited with their dams. In the AFR group, pups were not disturbed except for changing the bedding at P2, P10, $\mathrm{P} 17$, and P24. At P27, littermates were weaned and housed in groups of the same sex until 12 weeks old, at which offspring were transferred to a new cage for individual housing.

\section{Open field test and novel object recognition test}

Open field test was performed according to our previous report. ${ }^{12}$ Mice were placed on the center of an acrylic arena $(40 \times 40 \times 40 \mathrm{~cm})$ with a $10 \times 10 \mathrm{~cm}$ grid floor and allowed to freely investigate for 10 minutes. The level of lighting in the room was 100 lux. Behaviors of subject animals were recorded by a video camera on the ceiling board. The duration for which the subject was in the center area $(20 \times 20 \mathrm{~cm})$ was measured. The total path length was measured by counting the number of grids which a subject mouse walked over.

After the open field test, two objects of the same size $(3 \times 12 \times 1.5 \mathrm{~cm})$ were placed in the center area of the arena. Subjects were acclimated to the objects for 5 minutes. Then, one object was exchanged for a novel object $(6 \times 6 \times 5 \mathrm{~cm})$. Both novel and familiar objects were composed of black plastic cuboctahedrons connected by white arms but had different shape. Mice were again allowed to explore the objects for 5 minutes. Behaviors were videotaped, and video images were captured every 0.5 seconds. The duration that subjects sniffed at each object was calculated from the number of frames recording such behaviors. The novel object recognition index was calculated by dividing the duration that the mice sniffed the novel object by the sum of the total duration for sniffing each object. All apparatuses were cleaned with $70 \%$ ethanol after each test. Four mice (MS-WT, 1; AFR-KO, 2 ; and MS-KO, 1) were excluded from novel object recognition test analysis because of video recording issues.

\section{Marble burying test}

The marble burying test was performed with some modifications according to a previous report. ${ }^{25}$ Twenty-four glass marbles (12.5 $\mathrm{mm}$ in diameter, four rows of six marbles) were equally spaced on a $3 \mathrm{~cm}$ layer of wooden bedding material in a new cage $(30 \times 20 \times 13 \mathrm{~cm})$. Subjects were placed in the cage and allowed to explore the marbles for $30 \mathrm{~min}-$ utes under 70 lux. Behaviors were recorded by a video, and images were captured every 10 minutes. Captured images were binarized with the Yen threshold value, after subtracting the red component image from the blue component image using ImageJ software (National Institutes of Health [NIH]). The area of marbles in the subtracted image was measured. The number of marbles buried with bedding over $66.6 \%$ of their surface area was counted. Six mice (AFR-WT, 2; MS-WT, 1; AFR-KO, 2; and MS-KO, 1) were excluded from analysis due to escaping the apparatus or the failure of video imaging.

\section{Resident-intruder test}

The resident-intruder test was performed under the illumination of 100 lux as previously described. ${ }^{12}$ In brief, an intruder male mouse (WT, the same age and weight) was placed in the home cage of a subject mouse where the subject was kept for 3-5 days. Behaviors were videotaped for 5 minutes. The duration of behaviors by a subject was measured by counting the number of frames recording social behaviors after capturing video images every 0.5 seconds. Behaviors in response to the intruder were categorized as active (sniffing, chasing), passive (being sniffed, being chased), or aggressive (biting, kicking) behavior.

\section{Elevated plus maze test}

An elevated plus maze apparatus was placed under 52 lux lighting. ${ }^{12}$ A subject was placed at the center of the maze and allowed to explore for 10 minutes. The duration and number of entries onto the open arm were measured from video images recorded from the ceiling board. Five mice (AFR-WT, 2; 
MS-WT, 1; AFR-KO, 1; and MS-KO, 1) were excluded from analysis due to jumping off the apparatus.

\section{Three-chamber test}

The three-chamber test was carried out under the 100 lux lighting according to our previous report. ${ }^{12}$ A subject was acclimated to a white plastic apparatus $(30 \times 45 \times 25 \mathrm{~cm})$ containing two small stainless wire cages $(11 \mathrm{~cm}$ in diameter, $15 \mathrm{~cm}$ high) for 5 minutes. Then, the subject was allowed to explore the apparatus, in which one of the two wire cages contained an unfamiliar mouse (motopsin heterogenic, male, similar age with subject mouse) for 10 minutes. Another unfamiliar mouse was placed in the cage that was empty in the previous session. The subject mouse was again allowed to explore the apparatus, which contained a familiar and an unfamiliar mouse in each cage, for 10 minutes. Subjects were then returned to their home cage. Behaviors were videotaped, and the duration of sniffing cages was measured by counting the number of frames after capturing video images every 0.5 seconds.

\section{Immunohistochemistry}

Mice (AFR-WT, n=9; MS-WT, n=10; AFR-KO, n=11; and MS-KO, n=9) were sacrificed and transcardially perfused with PBS followed by 0.1 M phosphate buffer (PB, pH 7.4) containing $4 \%$ paraformaldehyde and $15.6 \%$ saturated picric acid, 3 hours after the three-chamber tests were completed. Brains were removed and post-fixed overnight in the same fixative solution, then soaked into $20 \%$ sucrose in $0.1 \mathrm{M} \mathrm{PB}$ ( $\mathrm{pH} 7.4$ ) at $4^{\circ} \mathrm{C}$ for a few weeks. Brains were coronally sliced at $35 \mu \mathrm{m}$ using a cryostat (CM3050S; Leica Microsystems, Wetzlar, Germany) and collected every $175 \mu \mathrm{m}$. Specimens were stored in cryoprotectant solution $(0.9 \% \mathrm{NaCl}, 30 \%$ sucrose, 1\% PVP-40, 30\% ethylene glycol, and 0.1 M PB, $\mathrm{pH} 7.4$ ) at $-20^{\circ} \mathrm{C}$ until use.

Immunohistochemistry was performed at room temperature as described previously. ${ }^{26,27}$ Sections were treated with PBS containing $6 \% \mathrm{H}_{2} \mathrm{O}_{2}$ and $30 \%$ methanol for 20 minutes to inactivate endogenous peroxidase after washing with PBS for 10 minutes, three times. Next, they were washed with PBS containing $0.3 \%$ Triton X-100 (PBS-T) three times and incubated overnight with rabbit anti-cFos antibody (SC-52; Santa Cruz Biotechnology Inc., Dallas, TX, USA, 1:1,000 dilution in PBS-T containing 1\% BSA). Sections were washed with PBS-T three times and then incubated with biotinylated antirabbit antibody (Vector Laboratories Inc., Burlingame, CA, USA, a 1:1,000 dilution in PBS-T containing 1\% BSA) for 2 hours. Sections were washed with PBS-T three times and then incubated with the complex of avidin and biotinylated horseradish peroxidase (Vector Laboratories Inc., 1:1,000 dilution in PBS-T) for 2 hours. To visualize immunoreactivity (IR), sections were reacted with $0.25 \mathrm{mg} / \mathrm{mL}$ diaminobenzidine and $0.01 \% \mathrm{H}_{2} \mathrm{O}_{2}$ in Tris- $\mathrm{HCl}(\mathrm{pH} 7.5)$, after washing with PBS-T three times. They were mounted onto glass slides (Matsunami Glass Ind., Ltd., Osaka, Japan).

Based on a mouse brain atlas, ${ }^{28}$ three sections around Bregma 1.54-1.94 were photographed using a microscope (ECLIPS 80i; Nikon Instruments, Melville, NY, USA) with a CCD camera (DFC290; Leica Microsystems). The quantification of cFos-positive cells was bilaterally performed in regions of interest (anterior cingulate cortex [AC], prelimbic cortex [PrL], infralimbic cortex [IL], 400×400 $\mu^{2}$ ) using ImageJ as previously described. ${ }^{26}$ The reasons that we analyzed in this region are the following. The number of cFospositive cells is reported to increase in these brain regions after social stimulations. ${ }^{26,29}$ Furthermore, the administration of MS paradigms disturbs the maturation of medial prefrontal cortex (mPFC) functions. ${ }^{18,30,31}$

\section{Statistics}

Statistical analysis was performed using IBM SPSS statistics software (version 24.0; IBM Corporation, Armonk, NY, USA). Outliers detected by box plots were excluded from statistical analysis. In order to analyze the effects of genotype (WT vs KO) and rearing condition (AFR vs MS) on behavioral tests and IR, two-way ANOVA was performed for each dependent variable within all samples using the Bonferroni adjustment for post hoc multiple comparisons. To analyze the time course in marble burying tests, the effects of time and group (AFR-WT, MS-WT, AFR-KO, and MS-KO) were analyzed by two-way ANOVA with repeated measure. All data are presented as mean \pm SEM. $P$-values of $<0.05$ were considered significant.

\section{Results \\ Open field test}

There were no significant effects or interaction on total path length during the open field test (Figure 2A, the main effect of genotype, $\mathrm{F}_{1,35}=0.063, P=0.804$; rearing condition, $\mathrm{F}_{1,35}=0.079, P=0.780$; the interaction between genotype and rearing condition, $\mathrm{F}_{1,35}=0.895, P=0.351$ ). Rearing condition, but not genotype, had a significant effect in terms of the duration in the center area (Figure 2B, the main effect of genotype, $\mathrm{F}_{1,33}=0.746, P=0.394$; rearing condition, $\mathrm{F}_{1,33}=5.060$, $P=0.031$; the interaction between genotype and rearing condition, $\left.\mathrm{F}_{1,33}=1.963, P=0.171\right)$. In the WT mice, the MS 

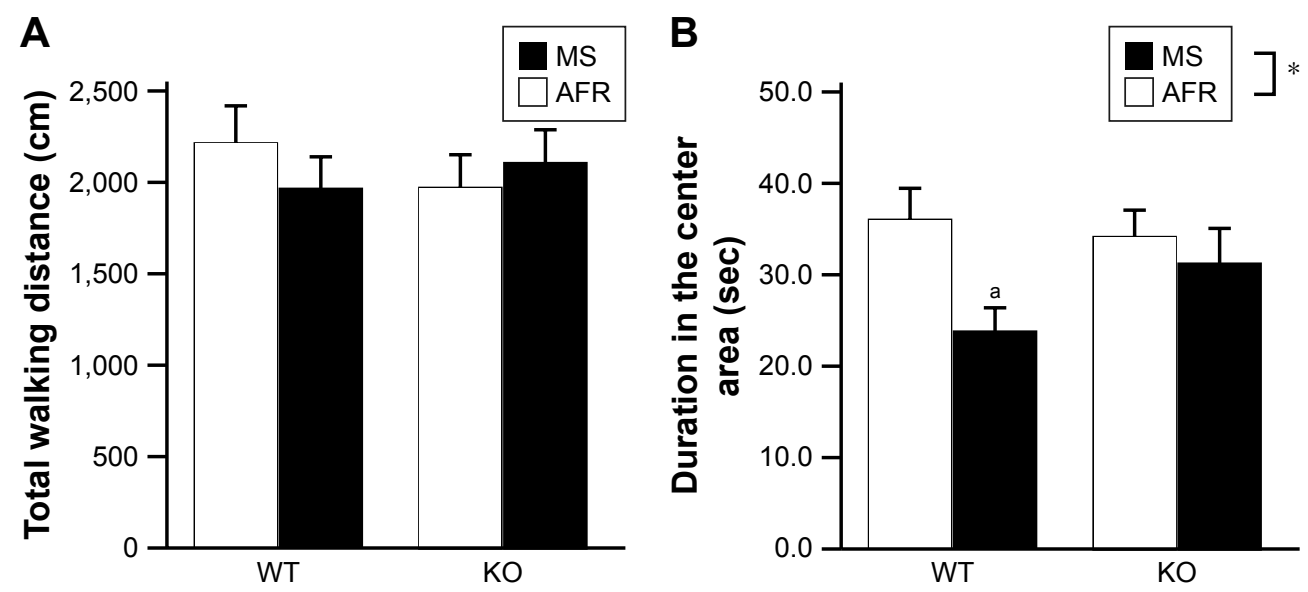

Figure 2 Rearing condition affected anxiety-like behavior in the open field test.

Notes: (A) There is no significant difference in total path length among the experimental groups. (B) MS-WT mice exhibited a decreased duration in the center area compared with AFR-WT mice. ${ }^{a} P<0.05$ vs AFR of the same genotype; $* p<0.05$.

Abbreviations: AFR, animal facility rearing; KO, knockout; MS, maternal separation; sec, seconds; WT, wild type.

paradigm decreased the duration in the center area $(P=0.015)$, suggesting increased anxiety. In contrast, in motopsin $\mathrm{KO}$ mice, the duration in the center area was not dependent on the rearing condition $(P=0.550)$.

\section{Novel object recognition test}

Statistical analyses revealed no significant effects on the total duration for investigating either the familiar or novel object (Figure 3A, the main effect of genotype, $\mathrm{F}_{1,35}=1.764, P=0.193$; rearing condition, $\mathrm{F}_{1,35}=0.951, P=0.336$; the interaction between genotype and rearing condition, $\mathrm{F}_{1,35}=0.506, P=0.481$ ). However, in terms of the novel object recognition index, the main effect of rearing condition was observed (Figure 3B, the main effect of genotype, $\mathrm{F}_{1,32}=0.253, P=0.618$; rearing condition, $\mathrm{F}_{1,32}=4.671, P=0.038$; interaction between genotype and rearing condition, $\mathrm{F}_{1,32}=0.276, P=0.603$ ). Post hoc analysis demonstrated a decreasing trend in the index with MS-KO mice compared with AFR-KO mice $(P=0.070)$, whereas such a trend was not detected with WT mice $(P=0.250)$.

\section{Marble burying test}

Two-way repeated ANOVA demonstrated significant effects of both group and time (Figure 4A, the main effect of the group, $\mathrm{F}_{3,1}=3.873, P=0.020$; time, $\mathrm{F}_{2.14,60.0}=1,115.012$, $P<0.001$; interaction between group and time, $\mathrm{F}_{6.42,60.0}=1.373$, $P=0.237)$. The number of buried marbles was increased in a time-dependent manner until 20 minutes for MS-KO mice, whereas there was a negligible increase in the number of buried marbles after 10 minutes in the other three groups. The statistical analysis revealed that WT mice buried significantly more marbles than $\mathrm{KO}$ mice at 10 minutes (Figure 4B, the main effect of genotype, $\mathrm{F}_{1,28}=6.885, P=0.014$; rearing condition, $\mathrm{F}_{1,28}=1.599, P=0.217$; interaction between genotype and rearing condition, $\mathrm{F}_{1,28}=0.392, P=0.536$ ), and this was similar at 30 minutes (Figure 4D, the main effect of genotype, $\mathrm{F}_{1,28}=3.773, P=0.062$; rearing condition, $\mathrm{F}_{1,28}=0.276, P=0.604$; interaction between genotype and rearing condition, $\mathrm{F}_{1,28}=3.049, P=0.092$ ). AFR-KO mice buried significantly fewer marbles than AFR-WT mice at all time points (Figure 4B-D; $P=0.030$ at 10 minutes, $P=0.037$ at 20 minutes, $P=0.015$ at 30 minutes).

\section{Resident-intruder test}

There was no significant effect or interaction on total contact time (Figure 5A, the main effect of genotype, $\mathrm{F}_{1,35}=0.972$, $P=0.331$; rearing condition, $\mathrm{F}_{1,35}=0.145, P=0.697$; interaction between genotype and rearing condition, $\mathrm{F}_{1,35}=0.118$, $P=0.733$ ), the duration of active behavior (Figure 5B, the main effect of genotype, $\mathrm{F}_{1,35}=0.215, P=0.646$; rearing condition, $\mathrm{F}_{1,35}=0.048, P=0.828$; interaction between genotype and rearing condition, $\mathrm{F}_{1,35}=0.971, P=0.331$ ), passive behavior (Figure 5C, the main effect of genotype, $\mathrm{F}_{1,35}=0.260, P=0.613$; rearing condition, $\mathrm{F}_{1,35}=1.9958, P=0.171$; interaction between genotype and rearing condition, $\mathrm{F}_{1,35}=1.440, P=0.238$ ), or aggressive behavior (Figure $5 \mathrm{D}$, the main effect of genotype, $\mathrm{F}_{1,35}=0.655, P=0.424$; rearing condition, $\mathrm{F}_{1,35}=0.033$, $P=0.856$; interaction between genotype and rearing condition, $\left.\mathrm{F}_{1,35}=1.021, P=0.319\right)$. The latency for the first contact was also comparable among experimental groups (Figure 5E, the main effect of genotype, $\mathrm{F}_{1,35}=0.032, P=0.859$; rearing condition, $\mathrm{F}_{1,35}=0.204, P=0.654$; interaction between genotype and rearing condition, $\left.\mathrm{F}_{1,35}=0.230, P=0.634\right)$. 


\section{A}

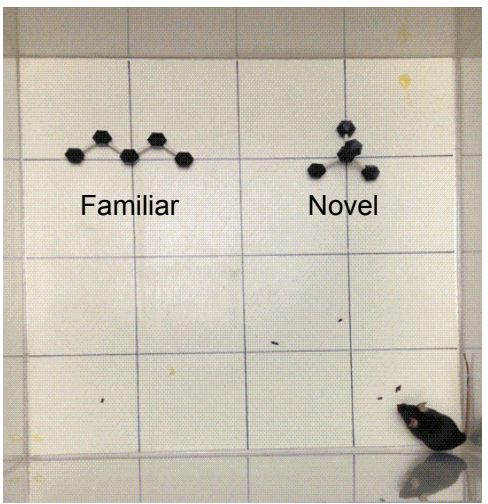

B

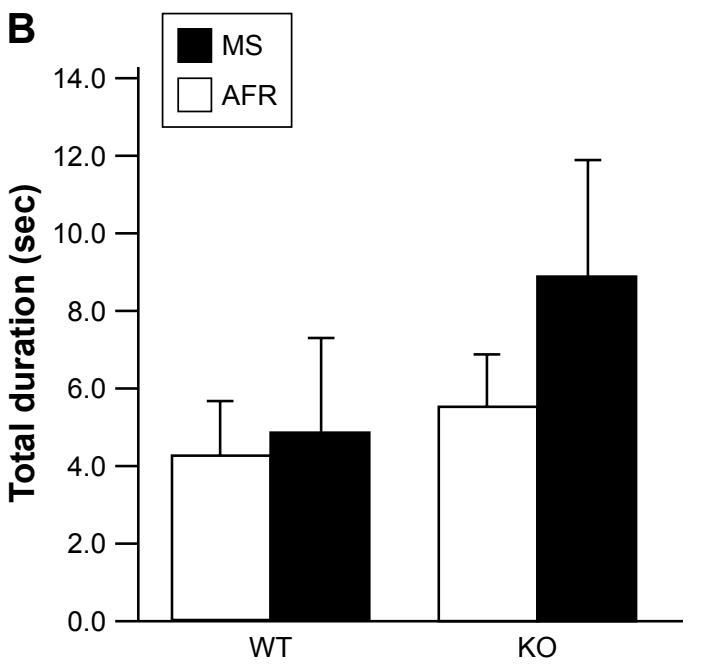

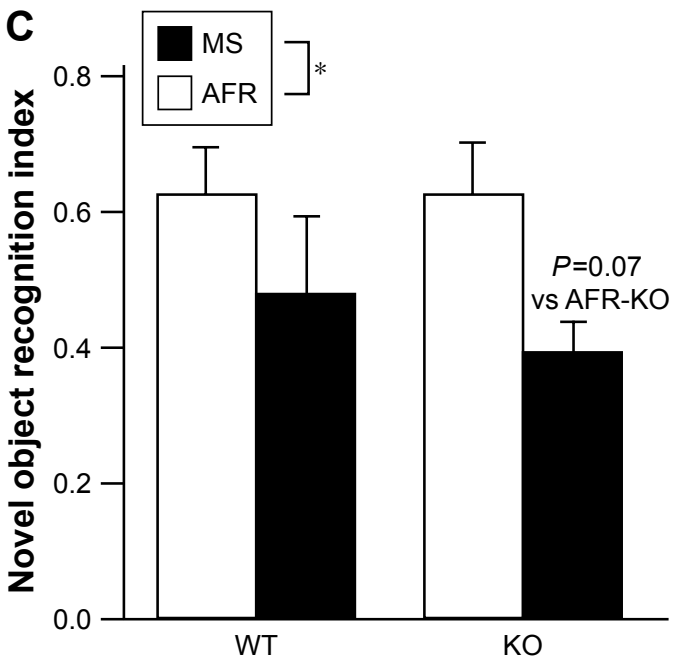

Figure 3 The MS paradigm decreased the desire to investigate a novel object.

Notes: (A) A novel and a familiar object was composed of five black cuboctahedrons and four arms. They were presented to a subject mouse for 5 minutes. (B) The total duration to investigate the objects was not significantly affected by rearing condition or genotype. (C) The novel object recognition index was reduced in MS mice. $* P<0.05$.

Abbreviations: AFR, animal facility rearing; KO, knockout; MS, maternal separation; sec, seconds; WT, wild type.

\section{Elevated plus maze test}

Although there was no significant effect or interaction on total arm entries (Figure 6A, the main effect of genotype, $\mathrm{F}_{1,36}=0.000, P=0.990$; rearing condition, $\mathrm{F}_{1,36}=0.536$, $P=0.469$; interaction between genotype and rearing condition, $\mathrm{F}_{1,36}=1.121, P=0.297$ ), in terms of the number of entries onto the open arm, there was a significant interaction between genotype and rearing condition (Figure 6B, the main effect of genotype, $\mathrm{F}_{1,33}=0.254, P=0.618$; rearing condition, $\mathrm{F}_{1,33}=0.000, P=0.989$; interaction between genotype and rearing condition, $\left.\mathrm{F}_{1,33}=5.176, P=0.030\right)$. AFR-KO mice entered open arms significantly more often than AFR-WT mice $(P=0.048)$. Interestingly, applying the MS paradigm tended to decrease the number of entries to the open arms for KO mice, but it increased the entries for WT mice; however, such trends were not significant in the post hoc analysis (KO, $P=0.121$; WT, $P=0.114$ ).
In terms of duration on open arms, no significant effects or interactions were found (Figure 6C, the main effect of genotype, $\mathrm{F}_{1,33}=0.158, P=0.693$; rearing condition, $\mathrm{F}_{1,33}=0.202$, $P=0.656$; interaction between genotype and rearing condition, $\mathrm{F}_{1,33}=1.052, P=0.312$ ).

\section{Three-chamber test}

In the first session in which the test apparatus contained an animal cage and an empty cage, no significant effects or interactions were detected on the total duration for investigating either cage (Figure 7A, the main effect of genotype, $\mathrm{F}_{1,35}=0.366, P=0.549$; rearing condition, $\mathrm{F}_{1,35}=0.126$, $P=0.725$; interaction between genotype and rearing condition, $\left.\mathrm{F}_{1,35}=0.000, P=0.988\right)$. To analyze the preference of the subjects, we calculated the proportion to sniff an animal cage by dividing the duration for sniffing an animal cage by the sum of durations to sniff an animal or empty cage. In terms 
A

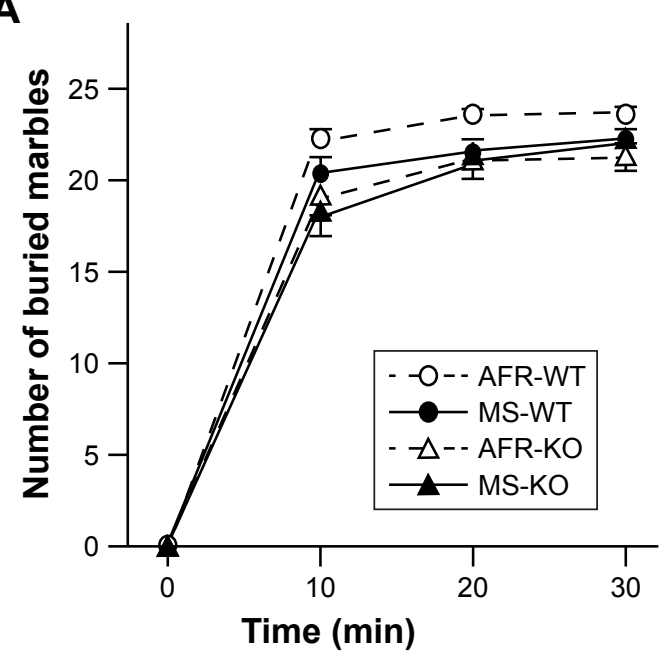

\section{C}

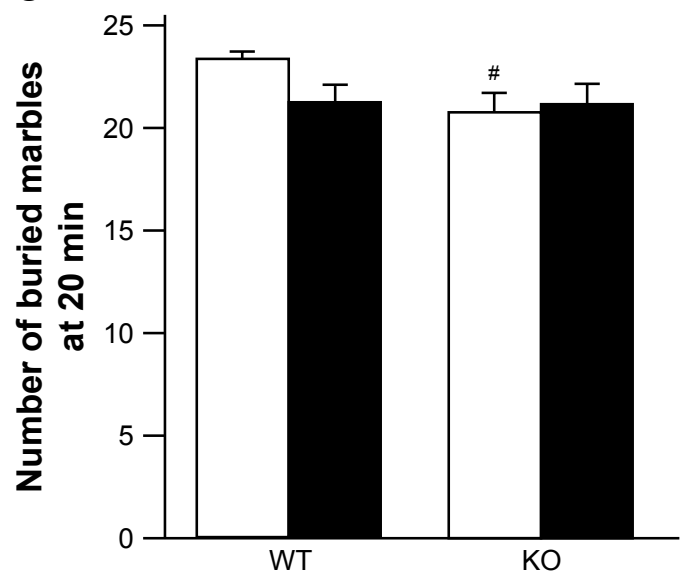

B

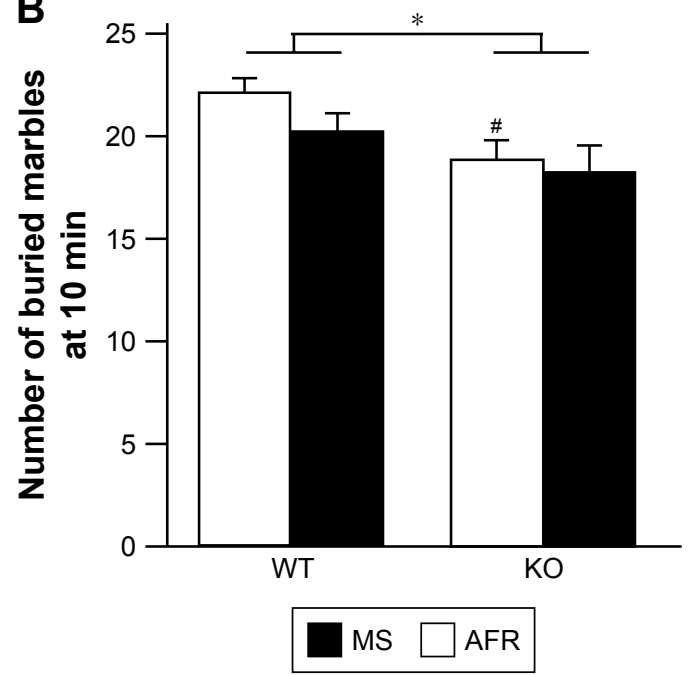

D

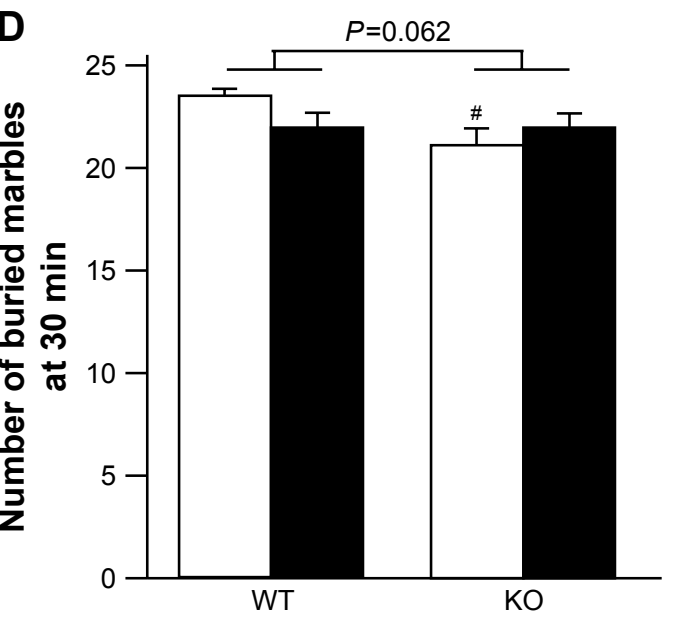

MS

Figure 4 Rearing condition and genotype affected perseverative behaviors.

Notes: (A) MS-KO mice buried marbles in a time-dependent manner. Other groups buried most of the marbles within the first 10 minutes and did not bury additional marbles afterward. (B) At 10 minutes, WT mice had buried more marbles than KO mice. (C) The number of buried marbles at 20 minutes. (D) WT mice tended to bury more marbles than KO mice, but this was not significant. ${ }^{\sharp} P<0.05$ vs WT mice of the same rearing condition; $* P<0.05$.

Abbreviations: AFR, animal facility rearing; KO, knockout; min, minutes; MS, maternal separation; WT, wild type.

of the proportion of the duration investigating the animal cage, there was a significant interaction between genotype and rearing condition (Figure $7 \mathrm{~B}$, the main effect of genotype, $\mathrm{F}_{1,35}=0.598, P=0.445$; rearing condition, $\mathrm{F}_{1,35}=0.539$, $P=0.468$; interaction between genotype and rearing condition, $\mathrm{F}_{1,35}=5.085, P=0.030$ ). In AFR conditions, motopsin KO mice exhibited a significantly reduced ratio sniffing the animal cage than WT mice $(P=0.037)$. Applying the MS paradigm significantly increased the ratio to investigate the animal cage for KO mice $(P=0.040)$, but it did not affect WT mice $(P=0.295)$.

In the second session of presenting a familiar and an unfamiliar mouse, statistical analyses revealed a significant interaction between genotype and treatment in terms of total duration to investigate stimulator mice (Figure $7 \mathrm{C}$, the main effect of genotype, $\mathrm{F}_{1,35}=1.809, P=0.187$; rearing condition, $\mathrm{F}_{1,35}=0.000, P=0.988$; interaction between genotype and rearing condition, $\left.\mathrm{F}_{1,35}=4.151, P=0.049\right)$. MS-KO mice demonstrated a significantly increased duration to sniff stimulator mice compared with MS-WT mice $(P=0.024)$. However, regarding the ratio of time investigating an unfamiliar mouse, no significant effects or interactions were observed (Figure 7D, the main effect of genotype, $\mathrm{F}_{1,35}=0.663$, $P=0.421$; rearing condition, $\mathrm{F}_{1,35}=1.659, P=0.206$; interaction between genotype and rearing condition, $\mathrm{F}_{1,35}=0.021$, $P=0.885)$. 

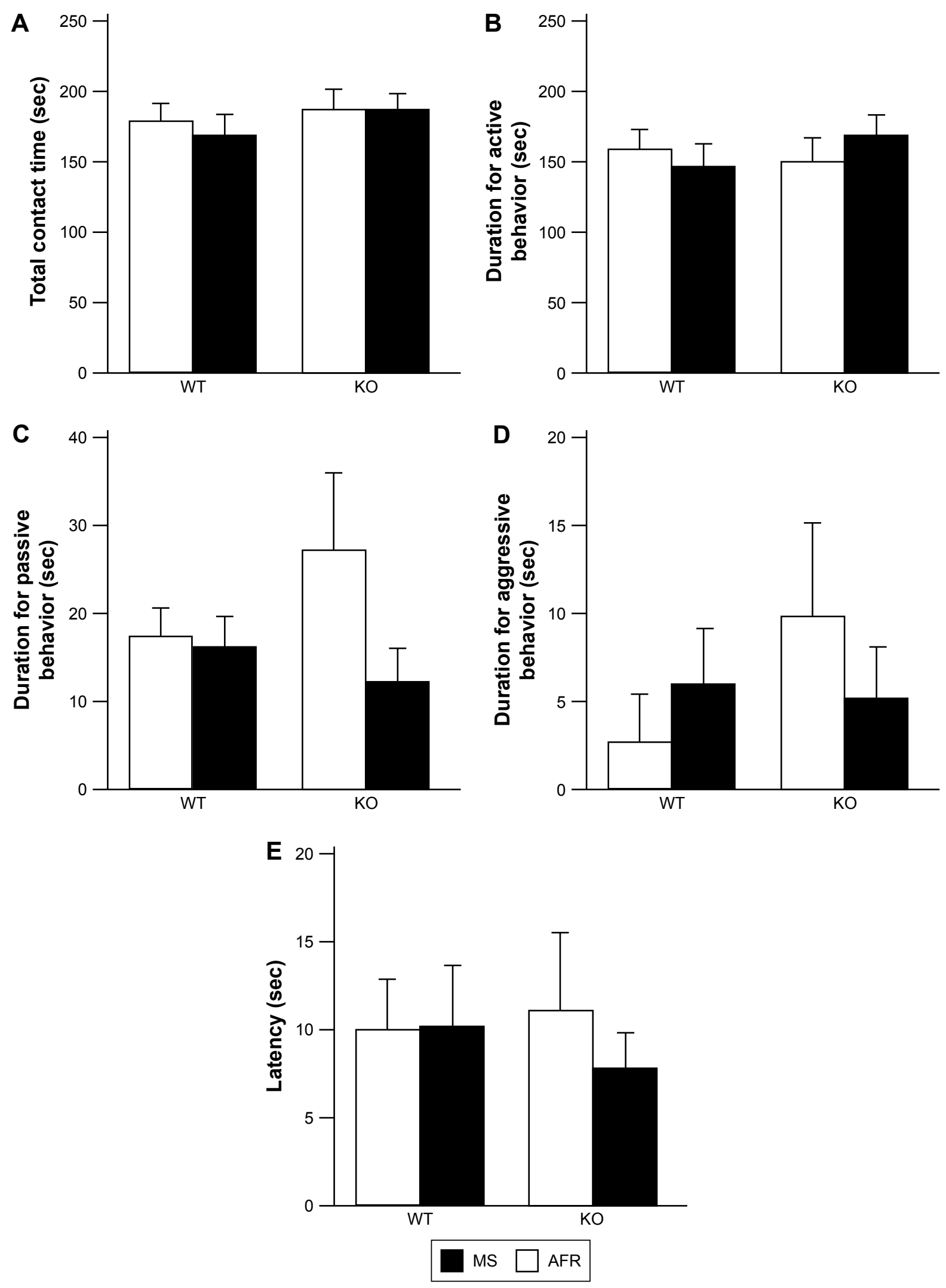

Figure 5 Neither rearing condition nor genotype affected social behaviors in the resident-intruder test.

Notes: (A) Total contact time. (B) The duration of active behaviors (chasing and anogenital sniffing). (C) The duration of passive behaviors (being chased, being anogenitally sniffed). (D) The duration of aggressive behaviors (biting, kicking). (E) The latency to the first contact.

Abbreviations: AFR, animal facility rearing; KO, knockout; MS, maternal separation; sec, seconds; WT, wild type. 

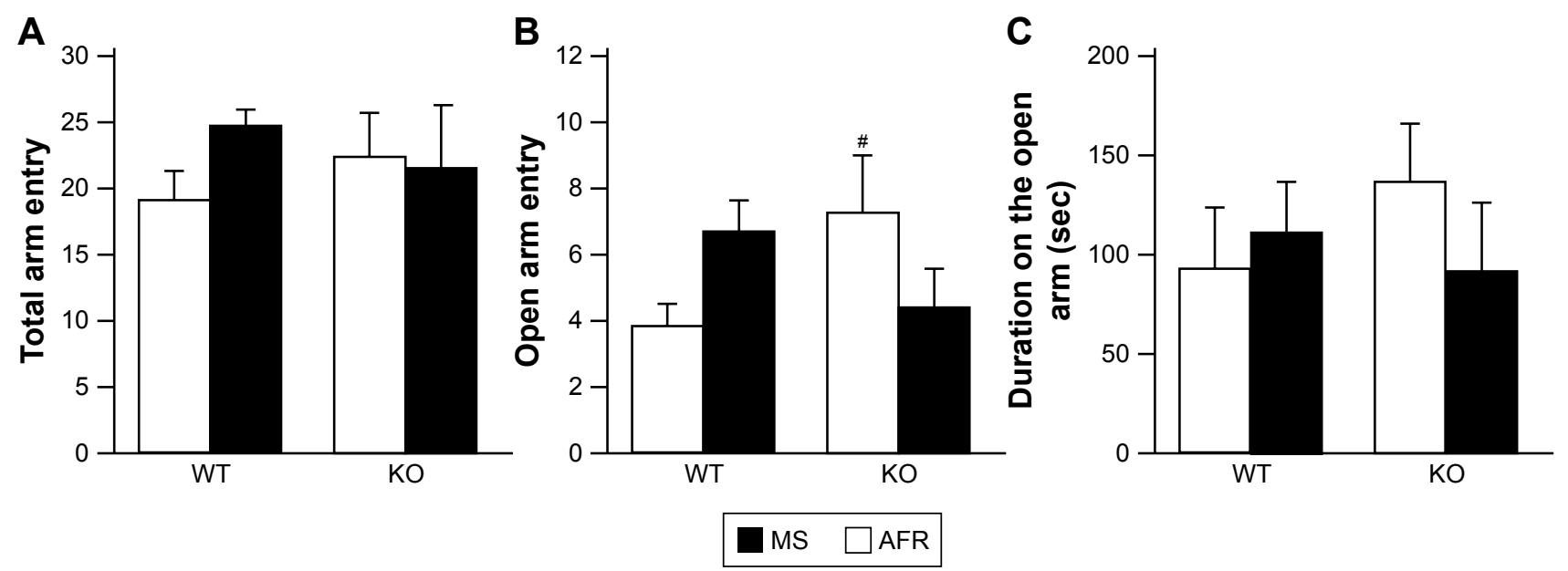

Figure 6 Rearing condition and genotype affected anxiety-like behavior in the elevated plus maze test.

Notes: (A) Total arm entries. There was no significant effect or interaction of genotype and rearing condition. (B) The number of open arm entries. The effects of MS were opposite between motopsin WT and KO mice. (C) The duration spent on the open arm. ${ }^{\sharp P}<0.05$ vs WT mice of the same rearing condition.

Abbreviations: AFR, animal facility rearing; KO, knockout; MS, maternal separation; sec, seconds; WT, wild type.

\section{cFos IR after social interaction}

At 3 hours after the second session of the three-chamber test, the number of cFos-positive cells in the mPFC was measured (Figure 8A-D). There was no significant difference in the AC (Figure 9A, the main effect of genotype, $\mathrm{F}_{1,31}=2.786, P=0.105$; rearing condition, $\mathrm{F}_{1,31}=0.096, P=0.759$; interaction between genotype and rearing condition, $\mathrm{F}_{1,31}=1.262, P=0.270$ ). The main effect of genotype was found on the PrL (Figure 9B, the main effect of genotype, $\mathrm{F}_{1,34}=8.708, P=0.006$; rearing condition, $\mathrm{F}_{1,34}=0.398, P=0.532$; interaction between genotype and rearing condition, $\mathrm{F}_{1,34}=1.137, P=0.294$ ), suggesting that motopsin deficiency decreases cFos-positive cells responding to social interactions. Post hoc analysis indicated that MS-KO mice had a decreased number of cFos-positive cells in the PrL compared with MS-WT mice $(P=0.009)$. Furthermore, two-way ANOVA revealed a significant interaction between genotype and rearing condition in the IL (Figure 9C, the main effect of genotype, $\mathrm{F}_{1,33}=3.582, P=0.067$; rearing condition, $\mathrm{F}_{1,33}=2.760, P=0.106$; interaction between effects of genotype and rearing condition, $\left.\mathrm{F}_{1,33}=4.914, P=0.034\right)$. In this region, MS significantly decreased the number of cFos-positive cells in KO mice $(P=0.012)$. MS-KO mice significantly showed fewer number of cFos-positive cells than MS-WT mice $(P=0.009)$.

\section{Discussion}

In order to clarify the effects of early-life adversity on adulthood behavior of subjects with atypical neurodevelopment, in this study, we applied the MS paradigm on motopsin KO mice. Effects of MS paradigms on adulthood behaviors are reported to vary among mouse strains, suggesting that genetic background contributes to MS effects. ${ }^{32}$ However, MS effects appear to be subtle because they are inconsistent among reports even in the same inbred strain. A systematic review revealed that increased, decreased, and unchanged levels of anxiety-like behaviors were reported in five $(35.7 \%)$, five (35.7\%), and four (28.6\%) papers, respectively, in C57BL/6 strain mice with MS treatment; the same strain has been used in this report. ${ }^{22}$ In this study, the MS paradigm raised anxietylike behavior in the open field test (Figure 2, the significant main effect of rearing condition). In particular, MS-WT mice spent a significantly shorter duration in the center area in the open field test, while the MS paradigm did not affect locomotor activity (Figure 2A).

The marble burying test is sometimes considered to be an evaluation of novelty-induced anxiety-like behavior because this test detects the efficacy of anxiolytic agents. ${ }^{25,33}$ Based on this, motopsin KO mice exhibiting less anxiety-like behavior than WT mice (the significant effect of genotype). AFR-KO mice buried significantly fewer marbles than AFR-WT mice. However, effects of the MS paradigm were not detected in this test. Together with the results from the elevated plus maze indicating a significant interaction but not the main effect of rearing conditions, it is likely that MS slightly increases anxiety-like behaviors in C57BL/6 WT mice in novel environments, such as an open field box, an elevated plus maze, or marbles, which they had never experienced until the current experiments.

Recent reports indicated that measurements from marble burying test do not correlate with open field test or light/dark 

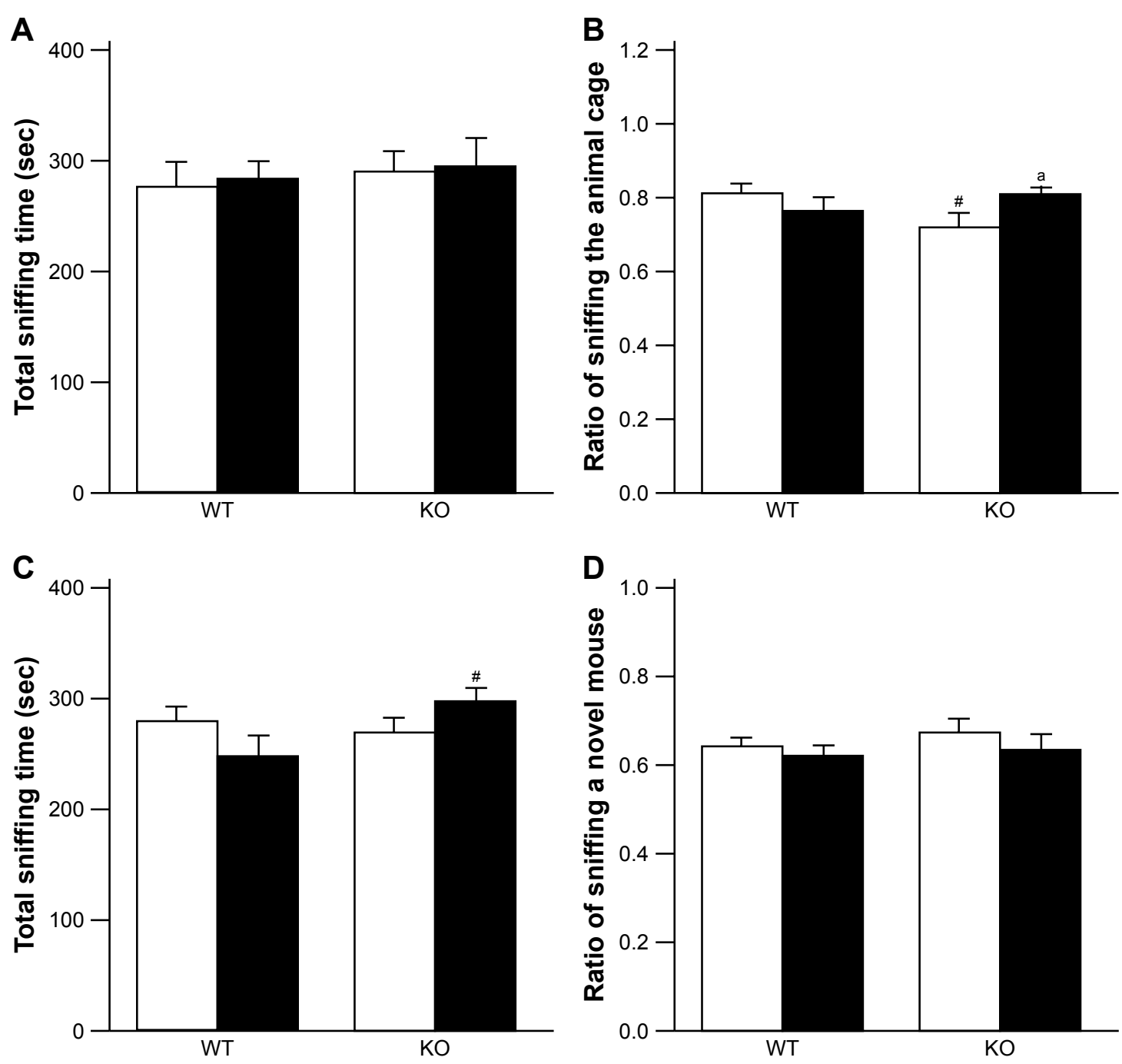

MS $\square$ AFR

Figure 7 Rearing condition and genotype affected social behaviors in the three-chamber test.

Notes: (A) Cumulative duration to investigate the empty or unfamiliar mouse-containing wire cage. (B) Motopsin deficiency reduced the ratio of sniffing the animal cage under AFR conditions, whereas MS increased it in only motopsin KO mice. (C) The MS paradigm increased the cumulative duration to sniff a cage containing a familiar or unfamiliar mouse by motopsin $\mathrm{KO}$ mice. (D) The ratio of investigating an unfamiliar mouse was not affected by rearing condition or genotype. ${ }^{a}<<0.05$ vs $A F R$ of the same genotype; ${ }^{\#}<0.05$ vs WT mice of the same rearing condition.

Abbreviations: AFR, animal facility rearing; KO, knockout; MS, maternal separation; sec, seconds; WT, wild type.

box test data, as indicated in this study. ${ }^{34,35}$ The number of buried marbles significantly correlates with repetitive digging behavior, which is affected by the familiarity of the bedding media. Marble burying is currently considered to reflect a kind of repetitive behavior. ${ }^{34,35}$ In this context, motopsin deficiency decreases perseverative behavior in a novel environment (the main effect of genotype). Time course analysis revealed that MS-KO mice buried marbles in time-dependent manner until 20 minutes, whereas AFR-KO mice buried negligible number of marbles after 10 minutes. The MS paradigm and motopsin deficiency may enhance each other.
The MS paradigm reduced the desire to explore a novel object in both motopsin KO and WT mice (Figure 3). As only motopsin KO mice tended to have a lower novel object recognition index by the MS paradigm, motopsin KO mice are more vulnerable to MS paradigms than WT mice. The longer time needed to bury marbles by MS-KO mice in the marble burying test may be attributed to the reduction of the impulse to explore novel objects.

The MS paradigm slightly affecting the social behavior of motopsin KO mice (Figures 5 and 7) was unexpected because motopsin deficiency increased the duration for exploring conspecifics in our previous report. ${ }^{12}$ Under AFR conditions, 
AFR-WT

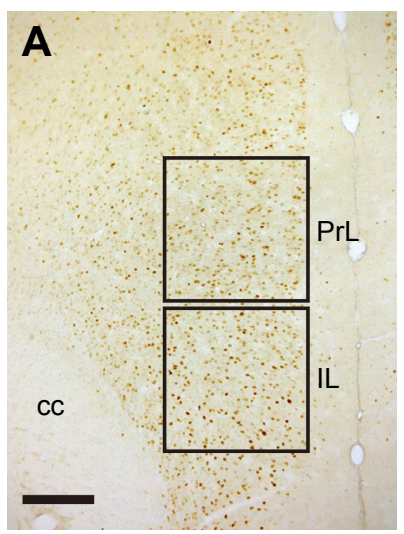

MS-WT

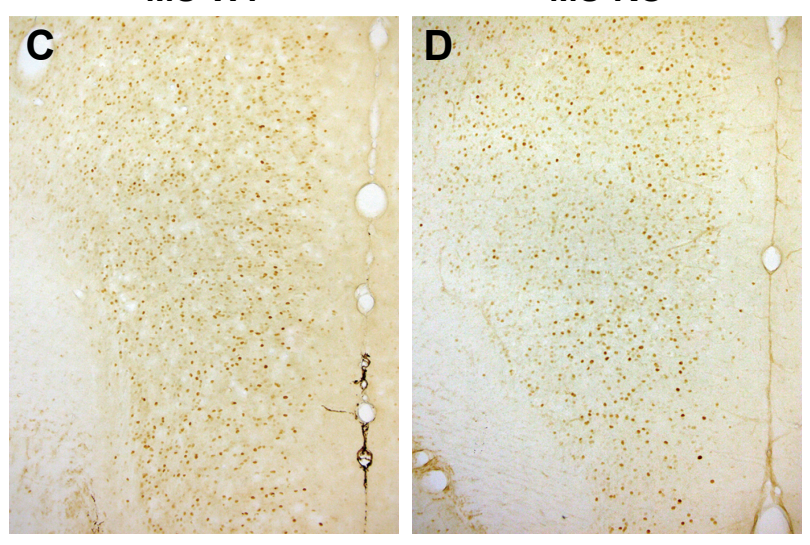

Figure 8 The immunoreactivity of cFos in the mPFC.

Notes: (A) AFR-WT. PrL and IL cortices are boxed. (B) AFR-KO. (C) MS-WT. (D) MS-KO. Bar, $200 \mu \mathrm{m}$.

Abbreviations: AFR, animal facility rearing; cc, corpus callosum; IL, infralimbic; KO, knockout; mPFC, medial prefrontal cortex; MS, maternal separation; PrL, prelimbic; WT, wild type.

motopsin $\mathrm{KO}$ mice had a decreased preference for sniffing an animal cage compared with WT mice, although MS-KO mice exhibited longer sniffing times compared with AFR-KO mice (Figure 7B). Such discrepancy between our previous and current reports may be derived from the difference in experimental conditions. Here, we performed residentintruder and three-chamber tests under light conditions (100 lux). In contrast, these tests were previously carried out under dim light conditions ( $<10$ lux), under which olfactory cues are more predominant than visual cues. The significant interaction between genotype and rearing condition means that the MS paradigm affects motopsin KO and WT mice in a different manner.

After social interaction in the three-chamber test, we measured the number of cFos-positive cells in the mPFC (Figures 8 and 9). The dorsal $\mathrm{mPFC}$, including the $\mathrm{AC}$, is believed to be related to motor function, having strong neuronal connectivity with the motor and pre-motor areas, and has been linked to several motor behaviors. ${ }^{36,37}$ The dorsal $\mathrm{mPFC}$ in rats also projects directly to the spinal cord. Neither rearing condition nor genotype affected the activation of the AC. This is consistent with the lack of significant effect of MS or genotype on total sniffing duration in the three-chamber test. The ventral mPFC, including PrL and IL, is strongly connected with limbic structures, including other regions of the limbic cortex, bed nucleus of the stria terminalis, nucleus accumbens, amygdala, and widespread regions of the hypothalamus and brain stem, whereas the dorsal mPFC essentially avoids connecting with these regions. The hippocampus projects to the IL/PrL but not to the AC. ${ }^{36}$ The ventral mPFC receives information from emotion-related structures and outputs adaptive emotional and autonomic responses. In the ventral $\mathrm{mPFC}$, the PrL is directly implicated in cognitive processes, and the IL markedly influences visceral/autonomic activities. ${ }^{36}$ No significant effects of MS were observed in the PrL, although motopsin KO mice had fewer cFos-positive cells than WT mice (Figure 9B), suggesting that motopsin deficiency impairs cognitive processes. This hypothesis is supported by our previous report that motopsin deficiency impairs hippocampal functions. In the IL, the MS paradigm decreased the number of cFos-positive cells in motopsin $\mathrm{KO}$ mice, but did not affect WT mice (Figure 9C), suggesting autosomal/visceral malfunction in MS-KO mice. Further investigations are needed to clarify the effects of the MS paradigm on visceral control in motopsin $\mathrm{KO}$ mice.

Inactivation of the ventral $\mathrm{mPFC}$ in motopsin $\mathrm{KO}$ mice suggests that motopsin deficiency disturbs the neuronal development of mPFC circuits and contributes to vulnerability to the MS paradigm, especially in circuits related to social contexts. Motopsin is a serine protease secreted from neuronal cells in an activity-dependent manner and induces dendritic filopodia, which is known to be an immature postsynaptic structure. ${ }^{9}$ The enhanced expression of motopsin in the cerebral cortex during the infantile period and the localization of this protease in the MPFC support this hypothesis. ${ }^{10,11}$ Moreover, overexpression of motopsin in neuronal cells enhances neurite formation, which is consistent with this idea. ${ }^{38}$

Motopsin deficiency causes severe intellectual disability in humans, ${ }^{6}$ although motopsin KO mice show comparable performance to WT mice in spatial memory tests and object recognition test. ${ }^{12}$ Intellectual disability in humans involves problems in the following two functions. One is an intellectual function, such as learning, problem solving, and judgment, and another is an adaptive function including activities of daily life, communications, and independent living. 

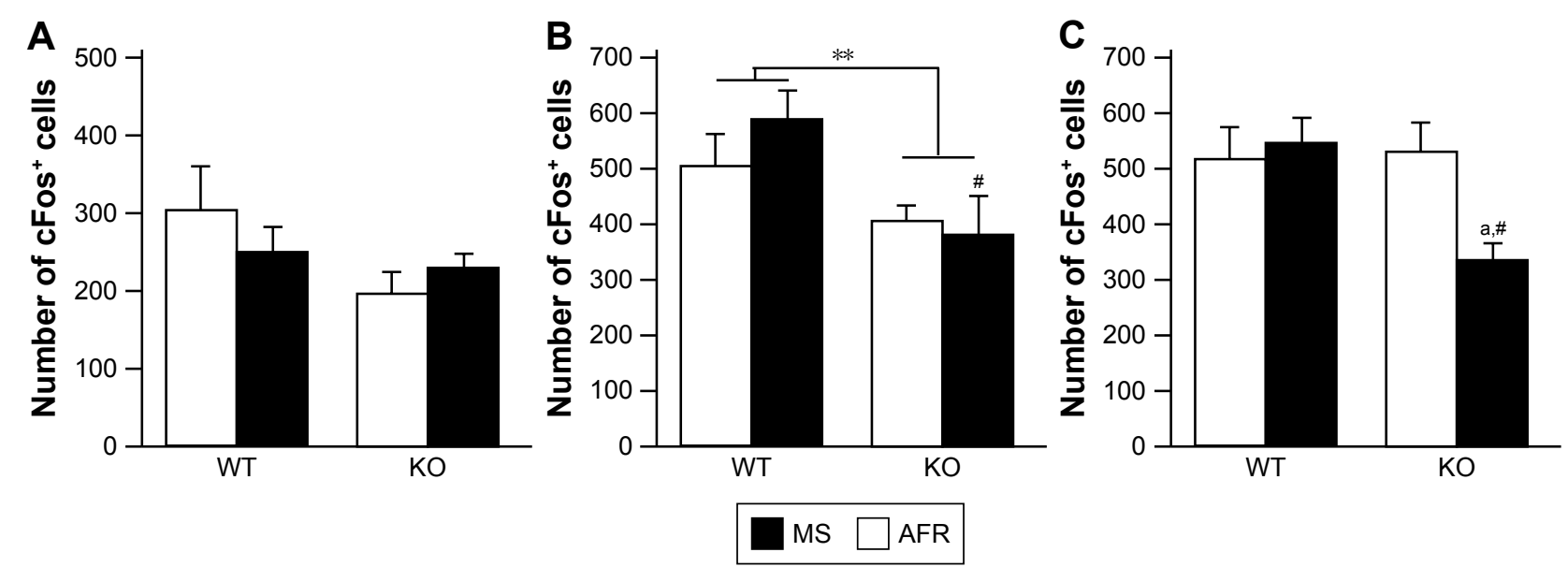

Figure 9 Rearing condition and genotype affected the number of cFos-positive cells in the mPFC after the three-chamber test.

Notes: (A) Anterior cingulate cortex. (B) Prelimbic cortex. (C) Infralimbic cortex. The number of cFos-positive cells was counted in $400 \times 400 \mu m^{2}$. ${ }^{P} P<0.05$ vs AFR of the same genotype; ${ }^{\# P<0.05}$ vs WT mice of the same rearing condition; $* * P<0.0$ I.

Abbreviations: AFR, animal facility rearing; KO, knockout; mPFC, medial prefrontal cortex; MS, maternal separation; WT, wild type.

However, the most behavioral deficit in mice is detected using simple tasks, such as an object recognition test in this study. These behavioral tests may be too simple to detect an intellectual disability in motopsin $\mathrm{KO}$ mice.

Behavioral data and cFos IRs in the mPFC are summarized in Table 1. Previous reports have indicated that MS impairs social behaviors and perturbs the functional maturation in $\mathrm{mPFC},{ }^{19,31}$ although the MS paradigm affected only open field and novel object recognition tests in this study. This inconsistency may be attributed to species and/ or experimental difference; rats vs mice, pups staying in group vs individual condition during the MS. However, there was a significant interaction between genotype and rearing condition in three examinations (elevated plus maze, three-chamber test, and cFos IR at the IL). This suggests that the MS paradigm affects motopsin KO and WT mice in different manners, probably because motopsin deficiency disturbs the development of neuronal circuits in the mPFC.

Table I Summary of the effects of rearing condition and motopsin deficiency on behaviors and PFC

\begin{tabular}{|c|c|c|c|c|c|c|c|}
\hline & \multicolumn{2}{|c|}{ Wild type } & \multicolumn{2}{|c|}{ Motopsin KO } & \multicolumn{3}{|c|}{ Significance of the effect } \\
\hline & AFR & MS & AFR & MS & Rearing & Genotype & Rearing *genotype \\
\hline \multicolumn{8}{|l|}{ Open field } \\
\hline Duration in the center & & $\theta$ & - & $\boldsymbol{r}$ & * & - & - \\
\hline \multicolumn{8}{|l|}{ Elevate plus maze } \\
\hline Open arm entry & & & & . & - & - & $*$ \\
\hline Open arm duration & - & -. & - & -. & - & - & - \\
\hline \multicolumn{8}{|l|}{ Marble burying } \\
\hline No of buried marbles & & & - & $\boldsymbol{r}$. & - & $*$ & - \\
\hline \multicolumn{8}{|l|}{ Novel object recognition } \\
\hline Novel object recognition index & & & & & $*$ & - & - \\
\hline \multicolumn{8}{|l|}{ Resident-intruder } \\
\hline Duration of active behavior & - & $\boldsymbol{r}$ & - & >. & - & - & - \\
\hline \multicolumn{8}{|l|}{3 Chamber test } \\
\hline Ratio of sniffing an animal cage & 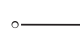 & $\boldsymbol{r}$ & & & - & - & $*$ \\
\hline Ratio of sniffing a novel mouse & - & -. & 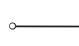 & $>$ & - & - & - \\
\hline \multicolumn{8}{|l|}{ cFos IR } \\
\hline$A C$ & - & -. & . & >. & - & - & - \\
\hline $\operatorname{PrL}$ & & & $\circ$ & >. & - & $* *$ & - \\
\hline IL & . & -. & & & - & - & $*$ \\
\hline
\end{tabular}

Notes: $* P<0.05 ; * * P<0.01 ;$ “-” indicates not significant.

Abbreviations: AC, anterior cingulate cortex; AFR, animal facility rearing; IL, infralimbic; IR, immunoreactivity; KO, knockout; MS, maternal separation; PFC, prefrontal cortex; PrL, prelimbic. 
Overall, it is likely that motopsin deficiency causes some behavioral impairment through disturbed development of neuronal circuits.

\section{Conclusion}

Although adversity during early life, such as MS, affects some adulthood behaviors, the effects depend on genetics in a complex manner. Concerning the motopsin gene, MS affected anxiety-like behaviors in WT mice but not in motopsin $\mathrm{KO}$ mice. In contrast, investigation of an animal cage in the three-chamber test was affected by the MS paradigm in motopsin KO mice, but not in WT mice. Furthermore, the MS paradigm appeared to deteriorate the impairment of motopsin $\mathrm{KO}$ mice in the marble burying test. Such complexity between the genetic background of motopsin and rearing conditions was observed in the cFos IRs at the mPFC. MS paradigms disturb neuronal circuits in broad brain areas, including the mPFC, hippocampus, and amygdala, all of which express motopsin. ${ }^{30,31,39}$ Identification of the comprehensive neuronal circuits that motopsin deficiency and MS paradigms modify is needed to understand the neuronal mechanisms underlying how adversity in early life affects motopsin $\mathrm{KO}$ mice.

\section{Acknowledgment}

This research was supported in part by Japan Society for the Promotion of Science (JSPS) KAKENHI grant No $17 \mathrm{~K} 10290$.

\section{Author contributions}

$\mathrm{CH}$ and $\mathrm{SM}$ conceived the project and designed the experiments. $\mathrm{CH}$ carried out most of the experiments. TK conducted the analysis of a resident-intruder test. DU performed the immunohistochemical analyses of cFos-positive cells in the mPFC. SM prepared the first draft of the manuscript, and all authors reviewed the draft manuscript, made revisions and approved the final draft for submission. All authors contributed toward data analysis, drafting and critically revising the paper and agree to be accountable for all aspects of the work.

\section{Disclosure}

The authors report no conflicts of interest in this work.

\section{References}

1. Halldorsdottir T, Binder EB. Gene x Environment Interactions: From Molecular Mechanisms to Behavior. Annu Rev Psychol. 2017;68(1): 215-241.
2. Lamblin M, Murawski C, Whittle S, Fornito A, Connectedness S. Mental health and the adolescent brain. Neurosci Biobehav Rev. 2017;80: 57-68.

3. Holz NE, Zohsel K, Laucht M, Banaschewski T, Hohmann S, Brandeis D. Gene $\mathrm{x}$ environment interactions in conduct disorder: Implications for future treatments. Neurosci Biobehav Rev. 2016;91:239-258.

4. Ropers HH. Genetics of intellectual disability. Curr Opin Genet Dev. 2008;18(3):241-250.

5. Mefford HC, Batshaw ML, Hoffman EP, Genomics HEP. Genomics, intellectual disability, and autism. $N$ Engl J Med. 2012;366(8):733-743.

6. Molinari F, Rio M, Meskenaite V, et al. Truncating neurotrypsin mutation in autosomal recessive nonsyndromic mental retardation. Science. 2002;298(5599):1779-1781.

7. Frischknecht R, Fejtova A, Viesti M, Stephan A, Sonderegger P. Activity-induced synaptic capture and exocytosis of the neuronal serine protease neurotrypsin. J Neurosci. 2008;28(7):1568-1579.

8. Gisler C, Lüscher D, Schätzle P, et al. Zymogen activation of neurotrypsin and neurotrypsin-dependent agrin cleavage on the cell surface are enhanced by glycosaminoglycans. Biochem J. 2013;453(1): 83-100.

9. Matsumoto-Miyai K, Sokolowska E, Zurlinden A, et al. Coincident preand postsynaptic activation induces dendritic filopodia via neurotrypsindependent agrin cleavage. Cell. 2009;136(6):1161-1171.

10. Iijima N, Tanaka M, Mitsui S, Yamamura Y, Yamaguchi N, Ibata Y. Expression of a serine protease (motopsin PRSS12) mRNA in the mouse brain: in situ hybridization histochemical study. Brain Res Mol Brain Res. 1999;66(1-2):141-149.

11. Mitsui S, Yamaguchi N, Osako Y, Yuri K. Enzymatic properties and localization of motopsin (PRSS12), a protease whose absence causes mental retardation. Brain Res. 2007;1136(1):1-12.

12. Mitsui S, Osako Y, Yokoi F, et al. A mental retardation gene, motopsin/ neurotrypsin/prss12, modulates hippocampal function and social interaction. Eur J Neurosci. 2009;30(12):2368-2378.

13. Nemeroff CB. Paradise Lost: The Neurobiological and Clinical Consequences of Child Abuse and Neglect. Neuron. 2016;89(5):892-909.

14. Nelson J, Klumparendt A, Doebler P, Ehring T. Childhood maltreatment and characteristics of adult depression: meta-analysis. Br J Psychiatry. 2017;210(2):96-104.

15. Mclaughlin KA, Sheridan MA, Nelson CA. Neglect as a Violation of Species-Expectant Experience: Neurodevelopmental Consequences. Biol Psychiatry. 2017;82(7):462-471.

16. Teicher MH, Samson JA, Anderson CM, Ohashi K. The effects of childhood maltreatment on brain structure, function and connectivity. Nat Rev Neurosci. 2016;17(10):652-666.

17. van Harmelen AL, van Tol MJ, van der Wee NJ, et al. Reduced medial prefrontal cortex volume in adults reporting childhood emotional maltreatment. Biol Psychiatry. 2010;68(9):832-838.

18. Proulx É, Suri D, Heximer SP, Vaidya VA, Lambe EK. Early stress prevents the potentiation of muscarinic excitation by calcium release in adult prefrontal cortex. Biol Psychiatry. 2014;76(4):315-323.

19. Yang $Y$, Cheng Z, Tang H, et al. Neonatal Maternal Separation Impairs Prefrontal Cortical Myelination and Cognitive Functions in Rats Through Activation of Wnt Signaling. Cereb Cortex. 2017;27(5):2871-2884.

20. Veenema AH, Bredewold R, Neumann ID. Opposite effects of maternal separation on intermale and maternal aggression in C57BL/6 mice: link to hypothalamic vasopressin and oxytocin immunoreactivity. Psychoneuroendocrinology. 2007;32(5):437-450.

21. Tractenberg SG, Levandowski ML, de Azeredo LA, et al. An overview of maternal separation effects on behavioural outcomes in mice: Evidence from a four-stage methodological systematic review. Neurosci Biobehav Rev. 2016;68:489-503.

22. Zoicas I, Neumann ID. Maternal separation facilitates extinction of social fear in adult male mice. Behav Brain Res. 2016;297: 323-328.

23. Nowak CB. Recognition and prevention of child abuse in the child with disability. Am J Med Genet C Semin Med Genet. 2015;169(4): 293-301. 
24. Wearick-Silva LE, Marshall P, Viola TW, et al. Running during adolescence rescues a maternal separation-induced memory impairment in female mice: Potential role of differential exon-specific BDNF expression. Dev Psychobiol. 2017;59(2):268-274.

25. Kedia S, Chattarji S. Marble burying as a test of the delayed anxiogenic effects of acute immobilisation stress in mice. J Neurosci Methods. 2014;233:150-154.

26. Nakamura K, Takabe A, Shimizu F, Takahashi M, Matsuo O, Mitsui S. Tissue plasminogen activator modulates emotion in a social context. Behav Brain Res. 2015;281:24-31.

27. Arai A, Hirota Y, Miyase N, et al. A single prolonged stress paradigm produces enduring impairments in social bonding in monogamous prairie voles. Behav Brain Res. 2016;315:83-93.

28. Franklin KBJ, Paxinos G. The Mouse Brain in Stereotaxic Coordinates. 3rd ed. NewYork: Elsevier; 2007.

29. Avale ME, Chabout J, Pons S, et al. Prefrontal nicotinic receptors control novel social interaction between mice. Faseb J. 2011;25(7): 2145-2155.

30. Soztutar E, Colak E, Ulupinar E. Gender- and anxiety level-dependent effects of perinatal stress exposure on medial prefrontal cortex. Exp Neurol. 2016;275 Pt 2:274-284.

31. Reincke SA, Hanganu-Opatz IL. Early-life stress impairs recognition memory and perturbs the functional maturation of prefrontalhippocampal-perirhinal networks. Sci Rep. 2017;7:42042.

32. Millstein RA, Holmes A. Effects of repeated maternal separation on anxiety- and depression-related phenotypes in different mouse strains. Neurosci Biobehav Rev. 2007;31(1):3-17.
33. Cryan JF, Sweeney FF. The age of anxiety: role of animal models of anxiolytic action in drug discovery. Br J Pharmacol. 2011;164(4): 1129-1161.

34. Thomas A, Burant A, Bui N, Graham D, Yuva-Paylor LA, Paylor R. Marble burying reflects a repetitive and perseverative behavior more than novelty-induced anxiety. Psychopharmacology. 2009;204(2): 361-373.

35. Wöhr M, Scattoni ML. Behavioural methods used in rodent models of autism spectrum disorders: current standards and new developments. Behav Brain Res. 2013;251:5-17.

36. Vertes RP. Interactions among the medial prefrontal cortex, hippocampus and midline thalamus in emotional and cognitive processing in the rat. Neuroscience. 2006;142(1):1-20.

37. Euston DR, Gruber AJ, Mcnaughton BL. The role of medial prefrontal cortex in memory and decision making. Neuron. 2012;76(6): 1057-1070.

38. Mitsui S, Hidaka C, Furihata M, Osako Y, Yuri K. A mental retardation gene, motopsin/prss12, modulates cell morphology by interaction with seizure-related gene 6. Biochem Biophys Res Commun. 2013;436(4): 638-644.

39. Nishi M, Horii-Hayashi N, Sasagawa T. Effects of early life adverse experiences on the brain: implications from maternal separation models in rodents. Front Neurosci. 2014;8:166.
Neuropsychiatric Disease and Treatment

\section{Publish your work in this journal}

Neuropsychiatric Disease and Treatment is an international, peerreviewed journal of clinical therapeutics and pharmacology focusing on concise rapid reporting of clinical or pre-clinical studies on a range of neuropsychiatric and neurological disorders. This journal is indexed on PubMed Central, the 'PsycINFO' database and CAS,

\section{Dovepress}

and is the official journal of The International Neuropsychiatric Association (INA). The manuscript management system is completely online and includes a very quick and fair peer-review system, which is all easy to use. Visit http://www.dovepress.com/testimonials.php to read real quotes from published authors. 